\title{
Clinical outcomes of transcatheter versus surgical pulmonary valve replacement: a meta-analysis
}

\author{
Ying Zhou, Tixiusi Xiong, Peng Bai, Chong Chu, Nianguo Dong \\ Department of Cardiovascular Surgery, Union Hospital, Tongji Medical College, Huazhong University of Science and Technology, Wuhan 430022, \\ China \\ Contributions: (I) Conception and design: C Chu, N Dong; (II) Administrative support: N Dong; (III) Provision of study materials or patients: \\ Y Zhou, C Chu, P Bai; (IV) Collection and assembly of data: Y Zhou, T Xiong, P Bai; (V) Data analysis and interpretation: Y Zhou, T Xiong, \\ C Chu; (VI) Manuscript writing: All authors; (VII) Final approval of manuscript: All authors. \\ Correspondence to: Chong Chu; Nianguo Dong. Department of Cardiovascular Surgery, Union Hospital, Tongji Medical College, Huazhong \\ University of Science and Technology, Wuhan 430022, China. Email: chongchu@hust.edu.cn; dongnianguo@hotmail.com.
}

Background: Transcatheter pulmonary valve replacement (TPVR) has currently been a well-established alternative operation method to surgical pulmonary valve replacement (SPVR) in patients with pulmonary valve dysfunction in the form of stenosis and/or regurgitation. We conducted a meta-analysis to evaluate the main clinical outcomes after TPVR and SPVR.

Methods: We systematically searched the references of relevant literatures from PubMed and the Cochrane Library published between January 2000 and December 2018 and followed The Preferred Reporting Items for Systematic reviews and Meta-analysis (PRISMA) for this study.

Results: Eleven studies with 4,364 patients were included in the study. Compared with SPVR, TPVR results in a significant decreased in-hospital mortality [odds ratio (OR): 0.18; 95\% confidence interval (CI): 0.03-0.98] and mortality at the longest reported follow-up time point (OR: 0.43; 95\% CI: 0.22-0.87), though 30-day mortality (OR: 0.38; 95\% CI: 0.11-1.33) has no significant difference between groups. Days of hospital stay [(mean difference (MD): -4.38; 95\% CI: -6.24--2.53] is shorter with TPVR than SPVR. Besides, rates of 30-day readmission (OR: 0.67; 95\% CI: 0.50-0.91) and recurrent pulmonary regurgitation (OR: 0.17; 95\% CI: 0.07-0.42) are lower with TPVR, whereas postprocedural infective endocarditis (IE) (OR: 4.56; 95\% CI: 2.03-10.26) are higher with TPVR. SPVR carries a decreased risk of re-operation (OR: 2.19; 95\% CI: 0.62-7.76) though without statistically significance.

Conclusions: In conclusion, TPVR is associated with a significantly decreased mortality, a shorter length of hospital-stay, a lower rate of 30-day readmission and recurrent pulmonary regurgitation as compared to SPVR throughout the follow-up duration, whereas SPVR results in a significantly lower rate of postprocedural IE than TPVR. In addition, SPVR carries a decreased risk of re-operation with statistically insignificance.

Keywords: Surgical pulmonary valve replacement (SPVR); transcatheter pulmonary valve replacement (TPVR); meta-analysis

Submitted Aug 07, 2019. Accepted for publication Nov 15, 2019.

doi: $10.21037 /$ jtd.2019.11.64

View this article at: http://dx.doi.org/10.21037/jtd.2019.11.64

(C) Journal of Thoracic Disease. All rights reserved. 


\section{Introduction}

Since Bonhoeffer et al. (1) initially described transcatheter pulmonary valve replacement (TPVR) for right ventricular outflow tract conduit dysfunction in the year 2000, more and more scholars have begun to study this technology and instruments, and TPVR is becoming a new interventional treatment and an emerging alternative to surgical pulmonary valve replacement (SPVR).

Transcatheter pulmonary valve replacement is designed for the treatment of circumferential right ventricular outflow tract (RVOT) conduit dysfunction when there is evidence of significant pulmonary stenosis and/or regurgitation. Indications of TPVR includes (2-4): (I) obvious right heart dysfunction after surgical repairment of complex congenital heart disease; (II) severe stenosis and/ or insufficiency of pulmonary valve after right ventricular outflow tract surgery; (III) absence of pulmonary valve; (IV) right ventricular outflow tract obstruction.

Compared with SPVR, TPVR is a minimally invasive surgical method newly developed, with advantages like minor surgical trauma, no need for general anesthesia and cardiopulmonary bypass support and avoiding median sternotomy (5).

Although the outcomes following TPVR have improved significantly in recent years, there is still controversy in the literature whether available data are robust enough to conclude that TPVR has better clinical outcomes like lower mortality and less postprocedural complications than SPVR. In this meta-study, we aimed to comprehensively evaluate the clinical outcomes of patients undergoing TPVR and SPVR in observational studies.

\section{Methods}

\section{Search strategy}

According to the Preferred Reporting Items for Systematic reviews and Meta-Analyses (PRISMA) amendment to the Quality of Reporting of Meta-analyses statement (6) and the recommendations from the Cochrane Collaboration and Meta-analysis of Observational Studies in Epidemiology (7), the study was carried out a systematical literature search in PubMed and the Cochrane Library published between January 2000 and December 2018 to identify eligible studies, using the search strings as follows: (pulmonary valve) AND (percutaneous OR transcatheter) AND (replacement OR implantation). Moreover, the reference lists of relevant articles and reviews were manually scrutinized to find additional studies that were eligible for inclusion.

\section{Eligibility criteria}

We included clinical studies only if they compared TPVR and SPVR and if they were designed as randomized and observational studies and published as original articles or abstracts. Studies that involved patients less than $30 \mathrm{~kg}$ in weight or with RVOT internal diameter less than $16 \mathrm{~mm}$ were not included in our analysis referring to the inclusion criteria for clinical trials with both the Melody and SAPIEN valves $(8,9)$, which are the mainly used bioprosthetic stentvalves in TPVR now. Diseased valves were replaced with only bioprosthetic valves in surgical procedure. Our primary outcome was all-cause mortality over the follow-up period. Articles that had not reported mortality were excluded. The secondary outcomes included length of hospital stay, 30-day readmission, recurrent pulmonary regurgitation, reoperation and postprocedural infective endocarditis (IE). Reviews, editorials, comments, expert opinions, duplicated publications, and non-English articles were excluded.

\section{Data extraction and quality assessment}

Two reviewers (Y Zhou and T Xiong) independently extracted data from studies, including study author, year, design, quality indicators, baseline clinical characteristics, procedural details, and clinical outcomes. Studies that met the inclusion criteria were rated based on the Newcastle Ottawa scale, with three main components, of study group selection, comparability between groups, and ascertainment of outcomes (10). A study with a NOS score of 6 or higher was regarded as of high quality. Any disagreements in data collection and quality evaluation were settled by the consensus between the two reviewers or a discussion with a third reviewer $(\mathrm{C} \mathrm{Chu})$.

\section{Statistical analysis}

In this study, categorical endpoints were presented as odds ratios (ORs) with corresponding 95\% confidence intervals (CIs) and continuous outcomes were presented as mean differences (MDs). Point estimates and standard errors were derived from each included study and were combined by the generic inverse variance method of DerSimonian and Laird (11). The random-effects model was prioritized in case of 


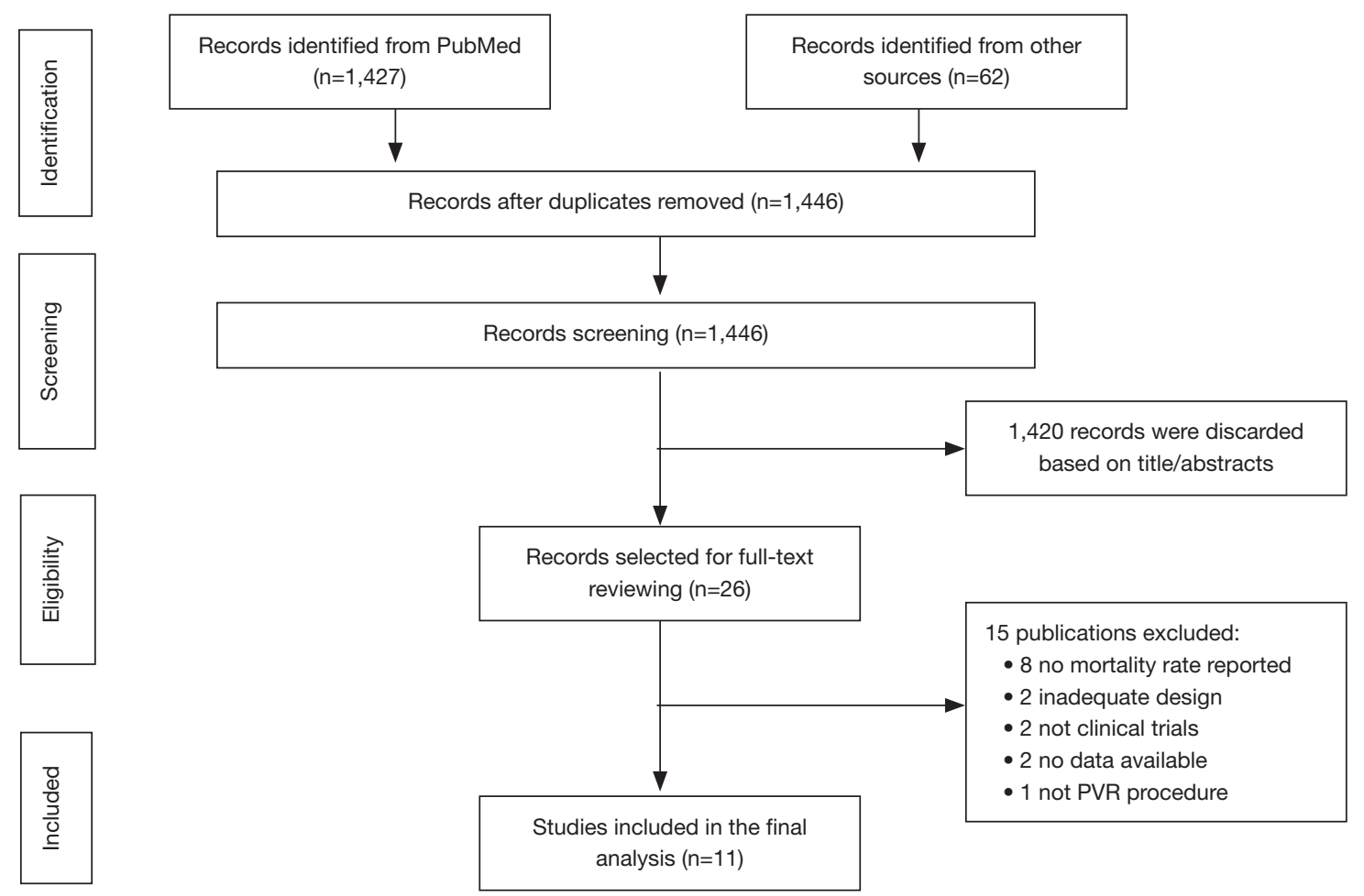

Figure 1 Flow diagram of study search process.

significant heterogeneity between studies; otherwise, the fixed-effects model was used.

Heterogeneity across studies was investigated by the Cochran $\mathrm{Q}$ test with a significant level of $\mathrm{P}<0.1$. In addition, we used the $\mathrm{I}^{2}$ statistic to quantify the heterogeneity, with an $\mathrm{I}^{2}$ value $>50 \%$ indicating substantial heterogeneity (12). Sensitivity analyses were performed by omitting each study in sequence. Publication bias was assessed by visual inspection of funnel plots and further confirmed by Harbord's test. All data analyses were carried out using the 'meta' package (13) in R (version 3.4.2, https:// www.R-project.org/). A two-sided $\mathrm{P}$ value of $<0.05$ was considered statistically significant.

\section{Results}

\section{Study search}

The study selection process was summarized in Figure 1. In general, of the initial 1,446 publications, 1,420 were excluded based on the titles and abstracts. The remaining 26 articles were selected for full-text reading, of which 15 reports that failed to meet the eligibility criteria were eliminated. Consequently, eleven observational studies (14-24) published between 2005 and 2018 were included in our meta-analysis. None meta-analysis comparing clinical outcomes between TPVR and SPVR has been previously reported.

All these 11 studies reported all-cause mortality data $(n=4,364), 4$ reported length of hospital stay $(n=556), 4$ reported 30-day readmission $(n=2,699), 5$ reported on patients with postprocedural re-pulmonary regurgitation $(\mathrm{n}=646), 5$ reported on patients undergoing re-operation $(n=2,615)$ and 6 reported on patients with postprocedural infective endocarditis $(n=1,137)$.

\section{Baseline characteristics}

The study characteristics were exhibited in Table S1. Briefly, all of the included studies were retrospective observational study, 2 of which were propensity-matched. Propensity score match is used to balance the distinction of observed baseline covariates and consequently enhance the comparability between groups (25). These eleven studies included data on a total of 4,364 patients with pulmonary valve disease, of which 1,284 patients receiving TPVR and 3,080 undergoing SPVR. Among the included studies, 8 
A. In hospital mortality

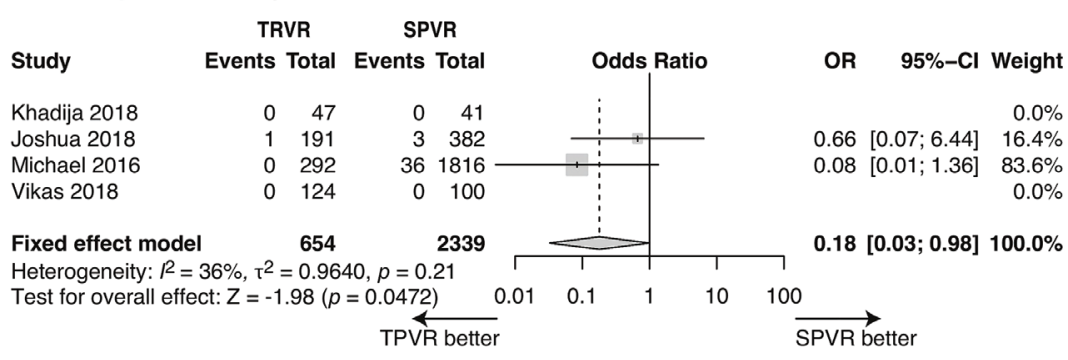

\section{B. 30 days mortality}

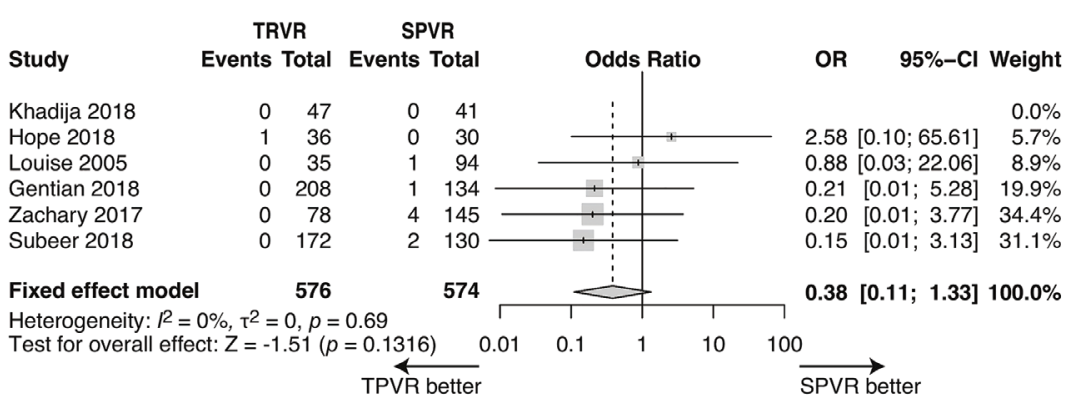

\section{C. the follow-up duration mortality}

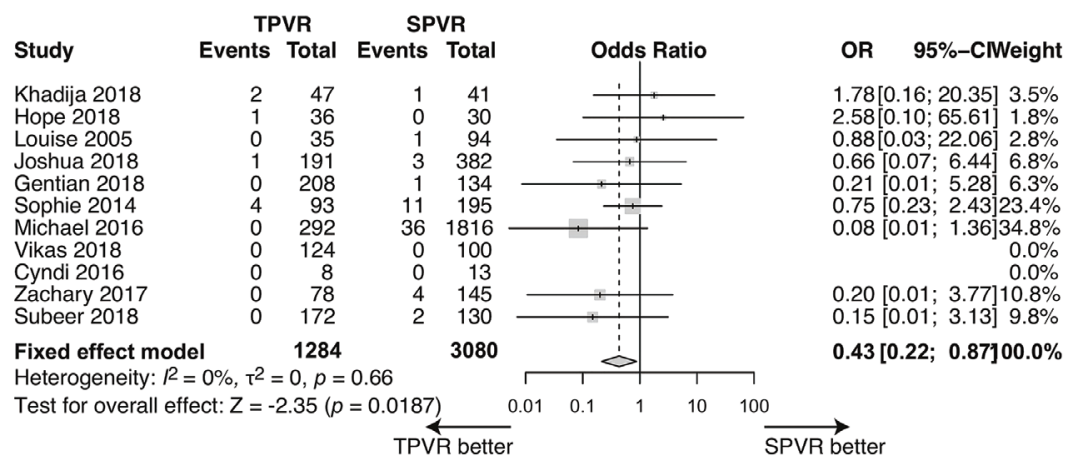

Figure 2 Meta-analysis for in-hospital, 30-day and throughout the follow-up duration mortality between TPVR vs. SPVR. TPVR, transcatheter pulmonary valve replacement; SPVR, surgical pulmonary valve replacement; CI, confidence interval.

were from the USA, 2 were from Europe, and the remaining 1 was conducted in Asia. The mean or median follow-up durations ranged from 2.2 to 89 months, the mean or median age ranged from 12 to 31.5 years, and men accounted for $59.6 \%$ of the total patients. The most commonly adjusted variables among the studies were age, sex, and primary cardiac diagnosis. Quality assessment showed a NOS score of 6 or higher for all studies with a mean NOS score of 6.8, indicating the presence of high methodological quality.

\section{All-cause mortality}

The all-cause mortality, calculated using data from 11 studies
( $n=4,364)$ was $0.62 \%$ in the TPVR group and $1.92 \%$ in the SPVR group. Compared with SPVR, TPVR procedure decreased the risk of death in hospital (4 retrospective cohort trails, $\mathrm{n}=2,993$, OR: $0.18,95 \%$ CI: $0.03-0.98, \mathrm{P}=0.047$, $\mathrm{I}^{2}=36 \%$, Figure $\left.2 A\right)$, at 30 days after procedure $(6$ retrospective cohort trails, $\mathrm{n}=1,150$, OR: $0.38,95 \%$ CI: $0.11-1.33, \mathrm{P}=0.13$, $\mathrm{I}^{2}=0 \%$, Figure $2 B$ ) and at the longest follow-up time point (11 retrospective cohort trails, $n=4,364$, OR: $0.43,95 \%$ CI: $0.22-0.87, \mathrm{P}=0.019, \mathrm{I}^{2}=0 \%$, Figure $2 C$ ).

\section{Length of hospital stay}

Four studies reported postoperative length of hospital stay. 


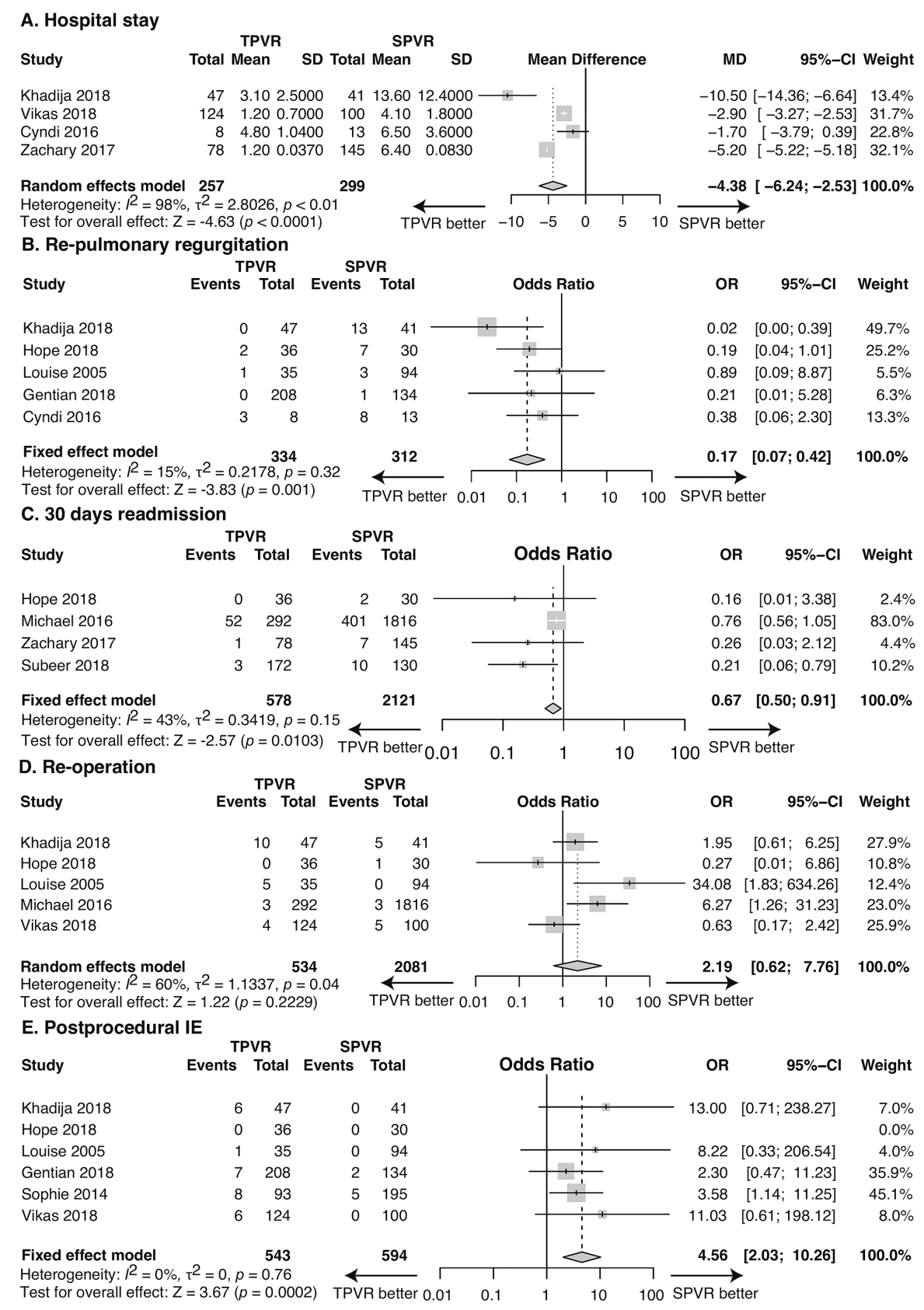

Figure 3 Meta-analysis for postoperative hospital stay and follow-up between TPVR vs. SPVR. TPVR, transcatheter pulmonary valve replacement; SPVR, surgical pulmonary valve replacement; CI, confidence interval.

With the length unit being converted to day, pooling the data of the included 556 patients in these 4 studies showed a significantly shorter length of hospital stay in the TPVR group than the SPVR group (MD: $-4.38,95 \% \mathrm{CI}:-6.24-$ $-2.53 ; \mathrm{P}<0.0001$, Figure $3 A$ ).

\section{Recurrent pulmonary regurgitation}

The recurrent pulmonary regurgitation rate was $1.80 \%$ in the TPVR group and $10.26 \%$ in the SPVR group, with no evidence of substantial heterogeneity across studies. 

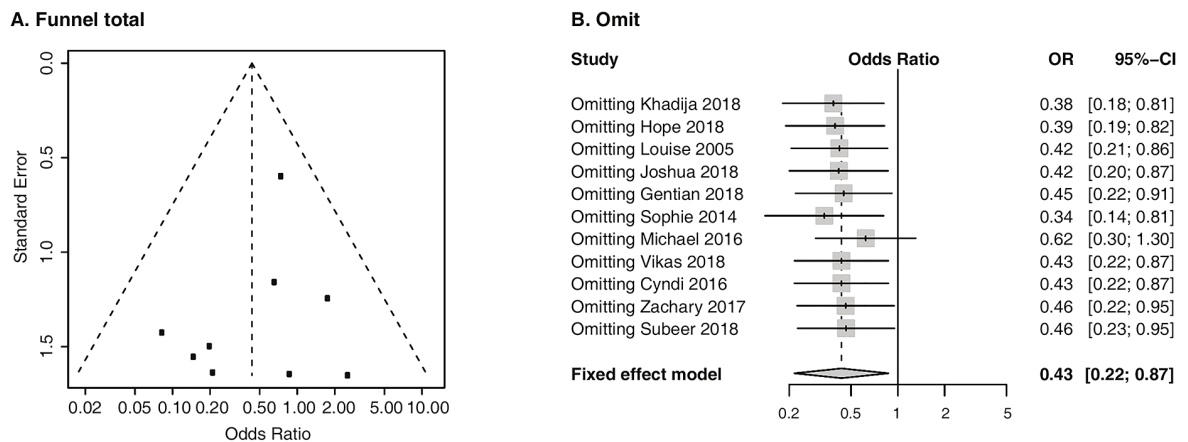

Figure 4 Funnel plots of mortality throughout the follow-up duration.

Compared with SPVR, TPVR was associated with an insignificantly decreased recurrent pulmonary regurgitation rate (5 retrospective cohort trails, $\mathrm{n}=646$, OR: $0.17,95 \%$ CI: $0.07-0.42, \mathrm{P}=0.001, \mathrm{I}^{2}=15 \%$, Figure $3 B$ ).

\section{0-day readmission}

Readmission within 30 days after procedure was observed in $9.68 \%$ and $19.80 \%$ of TPVR and SPVR recipients, respectively. The TPVR procedure was associated with significantly less 30-day readmission versus the SPVR procedure (4 retrospective cohort trails, $n=2,699$, OR: 0.67 , 95\% CI: $0.50-0.91, \mathrm{P}=0.010, \mathrm{I}^{2}=43 \%$, Figure 3 C).

\section{Re-operation}

Re-operation occurred in $4.12 \%$ of patients undergoing TPVR and $0.67 \%$ of patients undergoing SPVR. TPVR was associated with an insignificantly increased re-operation rate compared with SPVR (5 retrospective cohort trails, $\mathrm{n}=2,615$, OR: $2.19,95 \%$ CI: $0.62-7.76, \mathrm{P}=0.22, \mathrm{I}^{2}=60 \%$, Figure $3 D$ ).

\section{Postprocedural infective endocarditis}

Postprocedural infective endocarditis data were available from 6 trails $(\mathrm{n}=1,137)$. Infective endocarditis occurred in $5.16 \%$ of patients undergoing TPVR and $1.18 \%$ of patients undergoing SPVR. TPVR led to significantly increased postprocedural IE rate compared with SPVR (6 retrospective cohort trails, $n=1,137$, OR: $4.56,95 \%$ CI: $2.03-10.26, \mathrm{P}=0.0002, \mathrm{I}^{2}=0 \%$, Figure 3 E).

\section{Sensitivity analysis and publication bias}

All studies involved in our analysis that scored six or more on the Newcastle-Ottawa scale were included in sensitivity analysis. Figure $4 A$ shows a funnel plot of the studies included in this meta-analysis that reported the followup mortality. They all had even distributions around the vertical respectively, indicating the absence of publication bias. Exclusion of each study in sequence did not influence the overall results (Figure 4B). For other outcomes, the publication bias test was not performed due to the limited number of included studies.

\section{Discussion}

To our knowledge, this report presents the first metaanalysis to statistically integrate the heterogeneous results of individual studies and show evidence-based conclusions about major clinical outcomes of transcatheter versus surgical pulmonary valve replacement. The main findings of our study are as follows: (I) TPVR procedure resulted in lower all-cause mortality in hospital and at the longest follow-up time point versus SPVR procedure, although the between-group difference of postprocedural 30-day mortality was not statistically significant. (II) TPVR procedure was associated with much shorter postoperative length of hospital stay versus SPVR procedure. (III) TPVR procedure showed a lower incidence rate of recurrent pulmonary regurgitation and 30-day readmission but was trended towards a higher rate of postprocedural infective endocarditis and re-operation versus SPVR procedure.

With the surgical techniques and perioperative care of congenital heart diseases (CHD) developing rapidly, we are facing an increasing population of CHD patients who have lived to their adulthood now. These patients have undergone cardiovascular surgery in their childhood and the majority of them will require re-intervention in their adulthood (26) because of the hemodynamically significant 
right ventricular outflow tract (RVOT) dysfunction and pulmonary stenosis and/or regurgitation. Although cardiac surgery has always been the gold standard for treating pulmonary valve diseases, transcatheter approaches are growing to be more available and less invasive, which are especially suitable for increasingly complicated patients. The reported clinical outcomes vary substantially following SPVR and TPVR, obscuring rational guidance for clinicians, hospital administrators, and policy-makers. An overall comparison of clinical outcomes is urgently needed.

In the present meta-analysis, we found that transcatheter and surgical treatment of pulmonary valve replacement had rather low all-cause mortality throughout the followup period $(0.62 \%$ and $1.92 \%$, respectively), with TPVR procedure showing a better outcome. Considering the inconsistent follow-up time of the included studies, we performed a comparison of in-hospital mortality and 30-day mortality. Our data show a lower in-hospital and 30-day mortality of TPVR, though 30-day mortality lacks significant between-group differences. The likely explanation for this finding could be that TPVR is less invasive on account of a smaller incision, shorter procedural time and no need for cardiopulmonary bypass (CPB), and that subsequently reduces the potential ischemiareperfusion injury and inflammatory and arrhythmogenic effects caused by CPB $(22,24)$. As for 30-day mortality, perhaps a low enough mortality of SPVR at this time point makes it extremely difficult for TPVR to improve upon it.

Another important finding in our study is that TPVR was associated with much shorter hospital stay and lower incidence of 30-day readmission compared to SPVR procedure, which may be an important consideration for the younger generation. Earlier discharge and faster recovery time may be more preferable for young patients because it allows them to go back to school and daily activities as healthy kids earlier without falling behind (15).

It seems to be contradictory that SPVR had a higher rate of recurrent pulmonary regurgitation after the procedure whereas TPVR had a relatively higher reoperation rate, though both without statistical significance. In fact, both of these two operation methods have decent therapeutic efficacy. SPVR contains invasive procedures such as dissection the trunk of the pulmonary artery which may change the anatomical structures and result in a slightly higher rate of recurrent pulmonary regurgitation. Despite this fact, patients with postprocedural pulmonary regurgitation in SPVR group do not always need reoperation. Bioprosthetic valves in the pulmonary position are durable for a longer time and pulmonary valve regurgitation is usually well-tolerated through the midterm duration because the pulmonary valve is exposed to low pressure compared to the mitral or aortic valve. On the other hand, TPVR had a higher failure to implant rate due to inevitable 'learning curve' associated with the introduction of a new device and technique, or anatomic reasons such as predominantly excessively large RVOT or coronary or aortic root compression. Besides, complex postprocedural complications with TPVR also contributed to the higher re-operation rate. Common complications include stent fracture, endocarditis, paravalvular leak, thromboembolism, and valvular stenosis. During the procedure, hemodynamic collapse can occur secondary to hemorrhage from conduit rupture, coronary compression with resultant ischemia, or PA obstruction from valvular embolization (5).

Furthermore, we found that TPVR carries a rather higher rate of postprocedural infective endocarditis. In contrast with TPVR, SPVR may reduce the risk of infective endocarditis by removing previous valves or conduits, which are considered as a main source of infection (18). In addition, factors like friction between the valve and the conduit, incomplete apposition of the valve against the conduit and lack of valve reendothelialization may also contribute to the TPVR-related IE (14).

Though most of our findings are favorable for the transcatheter procedure, there remain restrictions when choosing TPVR. In the operation of TPVR, a large and stiff venous sheath and delivery system should be placed firstly, which can be difficult to maneuver into position from percutaneous approach in small children and/or patients with complex anatomy (27). Additionally, the majority of larger patients have severely dysfunctional RVOT's with internal diameter $>16 \mathrm{~mm}$, making it too difficult for safe and stable placement of the transcatheter valves. To overcome these restrictions, a hybrid approach has been proposed and successfully used (28). Surgeons adopted a subxiphoid incision to deliver a valve through a puncture in the right ventricular wall for smaller children. And for patients with enlarged RVOT's, a median sternotomy with creative ideas to reduce the RVOT size was used, including intravascular flow restrictors, extravascular radiopaque rings, and surgical plication. With these tricks, TPVR can have wider applications. Besides the above restrictions, patients with an active infection, coronary artery compression or aortic compression resulting in aortic insufficiency during pre-deployment balloon dilation and other unsatisfactory 
anatomy are very tricky and may turn to open surgical procedures for help (29).

Because of the rapid demographic changes of congenital heart disease, our findings are of great clinical importance. For patients who had previous CHD surgery and with inevitable re-intervention of PV dysfunction, our encouraging results confirmed the favorable findings for TPVR. Thus, TPVR appears safe and effective when compared to open surgery, allowing it to serve as an emerging alternative for many patients with PV dysfunction, especially for those who are of poor underlying conditions and intolerant to repeat redo cardiac surgery and the risk of myocardial injury related to repeated CPB. However, multi-center, randomized and prospective data are needed to better compare these two treatment modalities.

\section{Conclusions}

Taken together, our meta-analysis shows that TPVR is associated with significantly decreased mortality as compared to SPVR within in-hospital time and throughout the follow-up duration. Length of hospital stay is shorter following TPVR than SPVR. Rates of 30-day readmission and recurrent pulmonary regurgitation are lower with TPVR, whereas SPVR results in a significantly decreased risk of postprocedural IE. Besides, SPVR carries a decrease re-operation rate though without statistical significance. However, these findings should be considered within the current observation. Future larger studies, or perhaps randomized trials, are required to confirm the comparison of TPVR and SPVR.

\section{Limitations}

In common with all meta-analyses, its reliability is determined by the quality of the included references. Several limitations should be acknowledged in our work. Firstly, we included only observational studies due to the lack of RCT trials comparing TPVR and SPVR, which may increase the risk of selection bias and reduce the statistical power for some complications. Secondly, due to the lack of some individual patient-level data, we were unable to perform any subgroup analyses by some important confounders, such as indication for the procedure, concomitant surgical procedures, and prosthesis for replacement. Besides, it was impossible to perform an analysis to compare different approaches (transfemoral and transjugular) and other valverelated events such as bleeding and thrombosis due to lack of original data. Thirdly, the limited number of studies with longer follow-up time and the differences in endpoint definitions do not permit further inferences. Fourthly, there were significant heterogeneities in outcomes of length of hospital stay and re-operation which limited somewhat the interpretation of the results. Furthermore, data in the present meta-analysis were obtained from studies conducted mainly in the USA and Europe. Thus, generalization of our findings to other populations should be done with caution.

\section{Acknowledgments}

Funding: This work was supported by the National Key Research and Development Program of China [2016YFA0101103 to N Dong]; and the Scientific Research Training Program for Young Talents sponsored by Union Hospital, Tongji Medical College, Huazhong University of Science and Technology.

\section{Footnote}

Conflicts of Interest: The authors have no conflicts of interest to declare.

Ethical Statement: The authors are accountable for all aspects of the work in ensuring that questions related to the accuracy or integrity of any part of the work are appropriately investigated and resolved. This study was a secondary analysis regarding human subject data published in the public domain, thus no ethical approval was required.

\section{References}

1. Bonhoeffer P, Boudjemline Y, Saliba Z, et al. Percutaneous replacement of pulmonary valve in a right-ventricle to pulmonary-artery prosthetic conduit with valve dysfunction. Lancet 2000;356:1403-5.

2. Geva T. Indications and timing of pulmonary valve replacement after tetralogy of Fallot repair. Semin Thorac Cardiovasc Surg Pediatr Card Surg Annu 2006;9:11-22.

3. Oosterhof T, Hazekamp M, Mulder B. Opportunities in pulmonary valve replacement. Expert Rev Cardiovasc Ther 2009;7:1117-22.

4. Warnes CA, Williams RG, Bashore TM, et al. ACC/ AHA 2008 Guidelines for the Management of Adults with Congenital Heart Disease: Executive Summary: a report of the American College of Cardiology/American Heart Association Task Force on Practice Guidelines (writing 
committee to develop guidelines for the management of adults with congenital heart disease). Circulation 2008;118:2395-451.

5. Kheiwa A, Divanji P, Mahadevan VS. Transcatheter pulmonary valve implantation: will it replace surgical pulmonary valve replacement? Expert Rev Cardiovasc Ther 2018;16:197-207.

6. Moher D, Liberati A, Tetzlaff J, et al. Preferred reporting items for systematic reviews and meta-analyses: the PRISMA statement. BMJ 2009;339:b2535.

7. Stroup DF, Berlin JA, Morton SC, et al. Meta-analysis of observational studies in epidemiology: a proposal for reporting. Meta-analysis Of Observational Studies in Epidemiology (MOOSE) group. JAMA 2000;283:2008-12.

8. Alkashkari W, Alsubei A, Hijazi ZM. Transcatheter Pulmonary Valve Replacement: Current State of Art. Curr Cardiol Rep 2018;20:27.

9. Hascoet S, Acar P, Boudjemline Y. Transcatheter pulmonary valvulation: current indications and available devices. Arch Cardiovasc Dis 2014;107:625-34.

10. Wells GA, Shea B, O'Connell D, et al. The NewcastleOttawa Scale (NOS) for assessing the quality of nonrandomised studies in meta-analyses. Available online: http://www.ohri.ca/programs/clinical_epidemiology/ oxford.asp. 2014.

11. DerSimonian R, Laird N. Meta-analysis in clinical trials. Control Clin Trials 1986;7:177-88.

12. Higgins JP, Thompson SG. Quantifying heterogeneity in a meta-analysis. Stat Med 2002;21:1539-58.

13. Viechtbauer W. Conducting Meta-Analyses in $\mathrm{R}$ with the metafor Package. J Stat Softw 2010;36. doi: 10.18637/jss. v036.i03.

14. Alassas K, Mohty D, Clavel M, et al. transcatheter versus surgical valve replacement for a failed pulmonary homograft in the Ross population. J Thorac Cardiovasc Surg 2018;155:1434-44.

15. Caughron H, Kim D, Kamioka N, et al. Repeat Pulmonary Valve Replacement: Similar Intermediate-Term Outcomes With Surgical and Transcatheter Procedures. JACC Cardiovasc Interv 2018;11:2495-503.

16. Coats L, Tsang V, Khambadkone S, et al. The potential impact of percutaneous pulmonary valve stent implantation on right ventricular outflow tract re-intervention. Eur J Cardiothorac Surg 2005;27:536-43.

17. Daily JA, Tang X, Angtuaco M, et al. Transcatheter Versus Surgical Pulmonary Valve Replacement in Repaired Tetralogy of Fallot. Am J Cardiol 2018;122:498-504.
18. Lluri G, Levi DS, Miller E, et al. Incidence and outcome of infective endocarditis following percutaneous versus surgical pulmonary valve replacement. Catheter Cardiovasc Interv 2018;91:277-84.

19. Malekzadeh-Milani S, Ladouceur M, Iserin L, et al. Incidence and outcomes of right-sided endocarditis in patients with congenital heart disease after surgical or transcatheter pulmonary valve implantation. J Thorac Cardiovasc Surg 2014;148:2253-9.

20. O'Byrne ML, Gillespie MJ, Shinohara RT, et al. Cost comparison of Transcatheter and Operative Pulmonary Valve Replacement (from the Pediatric Health Information Systems Database). Am J Cardiol 2016;117:121-6.

21. Sharma V, Griffiths E, Eckhauser A, et al. Pulmonary Valve Replacement: A Single-Institution Comparison of Surgical and Transcatheter Valves. Ann Thorac Surg 2018;106:807-13.

22. Sosnowski C, Matella T, Fogg L, et al. Hybrid pulmonary artery plication followed by transcatheter pulmonary valve replacement: Comparison with surgical PVR. Catheter Cardiovasc Interv 2016;88:804-10.

23. Steinberg ZL, Jones TK, Verrier E, et al. Early outcomes in patients undergoing transcatheter versus surgical pulmonary valve replacement. Heart 2017;103:1455-60.

24. Wadia SK, Lluri G, Aboulhosn JK, et al. Ventricular arrhythmia burden after transcatheter versus surgical pulmonary valve replacement. Heart 2018;104:1791-6.

25. Deb S, Austin P, Tu J, et al. A Review of Propensity-Score Methods and Their Use in Cardiovascular Research. Can J Cardiol 2016;32:259-65.

26. Warnes CA, Liberthson R, Danielson GK, et al. Task force 1: the changing profile of congenital heart disease in adult life. J Am Coll Cardiol 2001;37:1170-5.

27. Bearl DW, Fleming GA. Utilizing Hybrid Techniques to Maximize Clinical Outcomes in Congenital Heart Disease. Curr Cardiol Rep 2017;19:72.

28. Agrawal H, Alkashkari W, Kenny D. Evolution of hybrid interventions for congenital heart disease. Expert Rev Cardiovasc Ther 2017;15:257-66.

29. Gales J, Krasuski RA, Fleming GA. Transcatheter Valve Replacement for Right-sided Valve Disease in Congenital Heart Patients. Prog Cardiovasc Dis 2018;61:347-59.

Cite this article as: Zhou Y, Xiong T, Bai P, Chu C, Dong N. Clinical outcomes of transcatheter versus surgical pulmonary valve replacement: a meta-analysis. J Thorac Dis 2019;11(12):53435351. doi: 10.21037/jtd.2019.11.64 


\section{Supplementary}

Table S1 Characteristics of the included studies

\begin{tabular}{|c|c|c|c|c|c|c|c|c|c|c|c|c|c|c|c|c|c|c|c|c|c|c|c|c|c|}
\hline \multirow{2}{*}{ Study author } & \multirow{2}{*}{ Publication year } & \multirow{2}{*}{ Study period } & \multirow{2}{*}{ Location } & \multirow{2}{*}{ Design } & \multirow{2}{*}{ NOS score- } & \multicolumn{2}{|c|}{ Sample size/n } & \multicolumn{2}{|c|}{ Follow-up time/m } & \multicolumn{2}{|c|}{ Age/yrs } & \multicolumn{2}{|c|}{ Gender, men $\% \%$} & \multicolumn{6}{|c|}{ Primary cardiac diagnosis/n } & \multicolumn{6}{|c|}{ Outcome parameters available for analysis } \\
\hline & & & & & & TPVR & SPVR & TPVR & SPVR & TPVR & SPVR & TPVR & SPVR & TOF & Ross procedure & TGA & Truncus arteriosus & DORV & Isolated PV disease & Mortality & Hospital stay & Readmission & Re-PR & Re-operation & Postprocedural IE \\
\hline Khadija et al. & 2018 & 1990-2015 & Saudi Arabia & $\mathrm{R}$, obs & 8 & 47 & 41 & 56 & 89 & 24 & 27 & 83 & 63 & 0 & 88 & 0 & 0 & 0 & 0 & + & + & & + & + & + \\
\hline Hope et al. & 2018 & Jan. 2007-Aug. 2017 & USA & $\mathrm{R}$, Obs & 7 & 36 & 30 & 25.8 & & $30.5^{\prime \prime}$ & 29.5" & 36.1 & 63.3 & 47 & 2 & 1 & NA & NA & $13^{t}$ & + & & + & + & + & + \\
\hline Louise et al. & 2005 & Oct. 1998-Mar. 2004 & UK & $\mathrm{R}$, obs & 6 & 35 & 94 & $4.0^{9}$ & $10.0^{\prime \prime}$ & Age $\mathrm{C}$ & & 45.7 & 67.0 & 83 & 6 & 12 & 7 & NA & $12^{4}$ & + & & & + & + & + \\
\hline Joshua et al. & 2018 & Jan. 2010-Dec. 2016 & USA & R, Obs, PM & 7 & 191 & 382 & 54 & & $17^{1}$ & $16^{n}$ & 58 & 62 & 573 & 0 & 0 & 0 & 0 & 0 & + & & & & & \\
\hline Gentian et al. & 2018 & Oct. 2010-Sep. 2016 & USA & R, Obs & 6 & 208 & 134 & $2.2^{n}$ & $2.8^{\prime \prime}$ & 25.7 & 23.3 & 57 & 54 & 184 & 26 & 14 & 30 & 28 & $48^{8}$ & + & & & + & & + \\
\hline Sophie et al. & 2014 & Jan. 2009-Jun. 2013 & France & $R$, obs & 7 & 93 & 195 & 24.1" & 23.8" & 20.1" & $13.3^{11}$ & 55.9 & 58.4 & 93 & 44 (AV disease) & 0 & 23 & & $12^{b}$ & + & & & & & + \\
\hline Michael et al. & 2016 & Jan. 2011-Dec. 2013 & USA & $R$, obs & 6 & 292 & 1816 & $\mathrm{NA}$ & $\mathrm{NA}$ & $17.7^{11}$ & $16.2^{n}$ & 62 & 61 & 297 & 0 & 2 & 10 & 69 & 18 & + & & + & & + & \\
\hline Vikas et al. & 2018 & Jan. 2010-Dec. 2015 & USA & $\mathrm{R}$, Obs & 7 & 124 & 100 & 18.7 & 31.6 & 19 & 12 & 58 & 54 & & & ggenital $f$ & RVOT disease (not s! & (ecific) & & + & + & & & + & + \\
\hline Cyndi et al. & 2016 & Jun. 2012-Jun. 2015 & USA & $\mathrm{R}$, obs & 7 & 8 & 13 & 3.4 & 13.6 & 31.5 & 31.3 & 50.0 & 61.5 & 14 & 0 & 0 & 0 & 0 & 7 & + & + & & + & & \\
\hline Zachary et al. & 2017 & Jan. 2006-Dec. 2013 & USA & $\mathrm{R}$, Obs, PM & 6 & 78 & 145 & $\mathrm{NA}$ & $\mathrm{NA}$ & 25.9 & 25.8 & 57.7 & 52.4 & 143 & 22 & 9 & 10 & 13 & 15 & + & + & + & & & \\
\hline Subeer et al. & 2018 & Oct. 2010-Dec. 2016 & USA & R, Obs & 8 & 172 & 130 & 15.6" & $12.0^{\circ}$ & $21.7^{11}$ & $21.0^{n}$ & 62 & 53 & 175 & 19 & $\mathrm{NA}$ & 21 & 17 & 344 & + & & + & & & \\
\hline
\end{tabular}

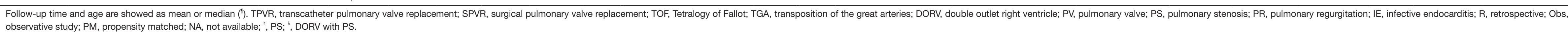

